\title{
MRI-guided high-intensity focused ultrasound as an alternative treatment option for multiple leiomyomas and huge leiomyomas
}

High-intensity focused ultrasound surgery is a potential non-invasive surgery for eliminating leiomyoma. In this short communication, we introduced an alternative role of this method for cases of multiple leiomyomas and huge leiomyomas with effective outcomes along with the pros and cons of these findings.

\section{KEYWORDS: multiple leiomyomas - huge leiomyoma - MRI-guided HIFU - non-invasive treatment}

\section{Introduction}

Uterine fibroids (known as leiomyoma) which disrupt the functions of the uterus and cause menorrhagia, dysmenorrhea, anemia, pelvic pressure or pain, urinary incontinence, recurrent pregnancy loss, and infertility, represent the most common tumor in women. The lifetime prevalence of fibroids is more than $80 \%$ among black women and about $70 \%$ among white women [1]. Patients with symptomatic multiple leiomyomas and huge leiomyoma desired to conserve the uterus for further delivery birth which generated one of the most common problematic medical conditions which have gynecologist needed to be considered and find the appropriate treatments cautiously.

Magnetic Resonance Imaging (MRI) guided High-intensity Focused Ultrasound (HIFU) which combines the anatomical and functional imaging of $\mathrm{MR}$ with the thermal ablation possibilities of HIFU, is an alternative treatment to hysterectomy for the treatment of uterine fibroids and preserves the uterus [2,3]. In this case report, we aimed to display one tough case of 15 leiomyomas and another tough case of huge leiomyoma ablated profitably by single phase HIFU along with the pros and cons of these findings.

\section{Methods}

By virtue of focusing one energy source to one targeted point, the heating will appear (FIGURE 1). The production of thermal energy by means of HIFU manages both the ablative and hyperthermal consequences (FIGURE 2). In each circumstance, the mechanisms of heat creation are identical,

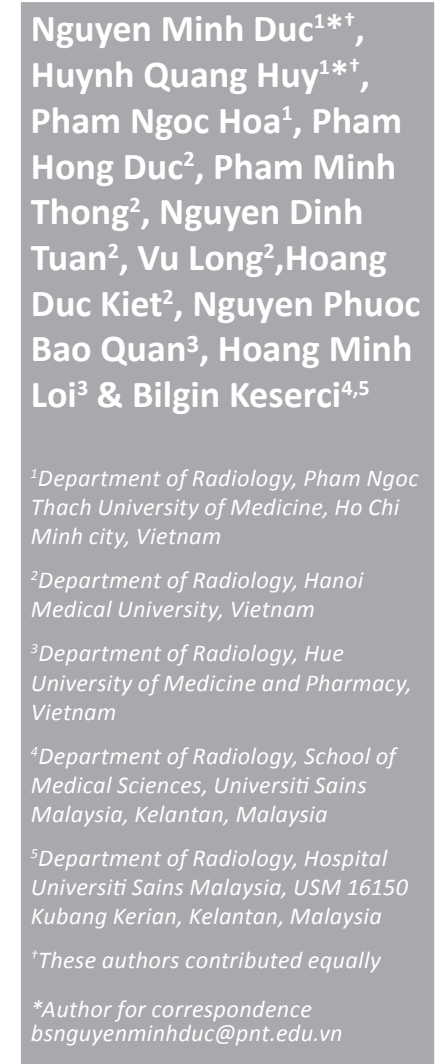

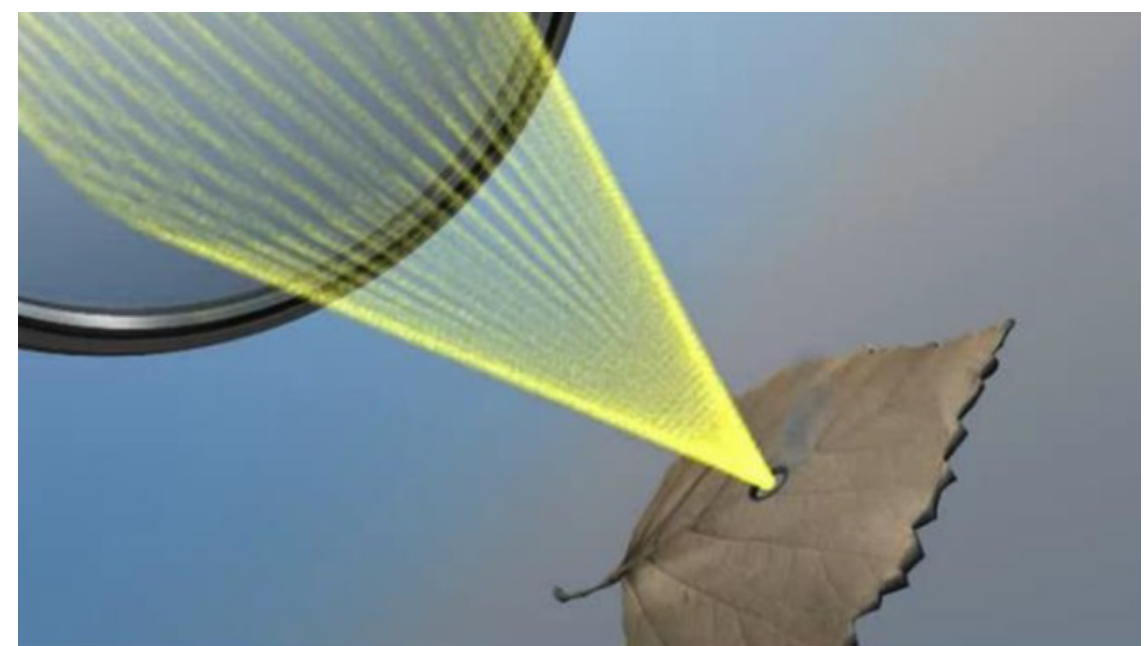

Figure 1. The basic principle of high-intensity focused energy at a targeted point. 


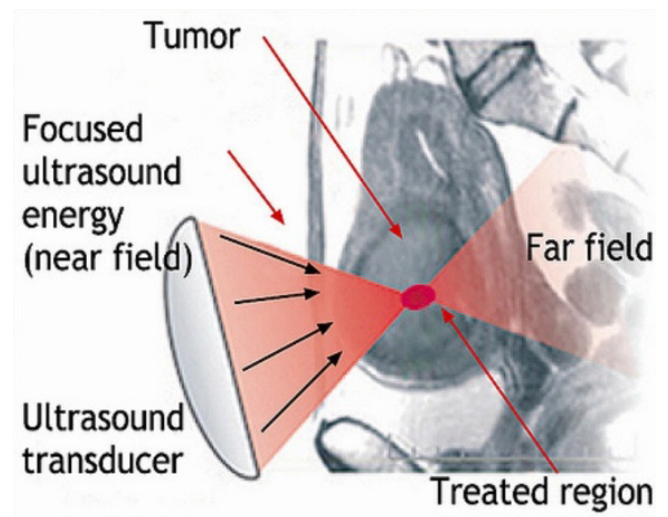

Figure 2. A schematic image manifests the principle of HIFU at a targeted uterine leiomyoma.

solely its level is dissimilar. Tissue temperature can be elevated up to the threshold $\geq 56^{\circ} \mathrm{C}$ for protein degeneration; thus manufacturing tissue necrosis. When non-linear ultrasonic waves pass through tissue, the difference of pressure leads to produce microscopic cavitation and heat accumulation inside the tissue. At adequate amplitudes, gaseous cavities appear, with the consequent performance of these being divided into two classes: stable and unstable. Stable cavitation regards as sustained oscillation of the intact bubble, whereas unstable cavitation is an accelerated extension of the cavity with a subsequent rupture, which is along with the concentrated acoustic power discharge. Heating takes place via both the absorption of acoustic energy and discharge of ultrasonic power inside the cavitation, causing a targeted deposition of the acoustic energy. It is acknowledged that as single frequency ultrasonic waves propagate an inharmonious environment, the energy will be scattered, dispersed and absorbed by surrounding tissues in front of the final targeted point. Therefore, the remaining ultrasonic power at the focused point is reliant on the magnitude of the wave, tissue characteristics of final point and surroundings and distance from the transducer to target. These factors are substantial contributors to thermal generation and efficacy in HIFU field. HIFU thermal outcomes such as protein degeneration and tissue coagulation necrosis consistently appear at a temperature of $\geq 43^{\circ} \mathrm{C}$ with an exposure length of at least $1 \mathrm{~h}$ whereas rapidly occur at the temperature of $56^{\circ} \mathrm{C}$ with an exposure length of only 1 second $[4,5]$.

There are currently two real-time modalities guided HIFU: ultrasonography and MRI.
There are some pros and cons between these methods. Firstly, in terms of space-consuming, noisy, bulky, positioning, procedure related to anesthesia and investment budget, ultrasoundguided HIFU is superior to MRI-guided HIFU. On the other hand, in terms of, contrast quality of real-time image, quantitative data for research and the precise information of temperature mapping, MRI-guided HIFU is predominant to ultrasound-guided HIFU. In detail, ultrasonography-guided HIFU has a comparably low spatial resolution, hence limits its precision for targeting and visualizing the details of the adjacent structures or organs. MRI offers a real-time thermal resolution with high spatial resolution, thus allows detection of small temperature elevations outside the targeted point prior to the appearance of irreversible tissue damage [6,7].

\section{Results}

A nulliparous 30 y old patient with history of uterine leiomyoma complained bulk effect of pelvis admitted to department of gynecology. MRI examination revealed that there were 15 uterine leiomyomas affected almost areas of anteflexed uterus considered as all type II uterine leiomyomas (the signal intensity of leiomyoma was greater than that of skeletal muscle and less than that of myometrium) (FIGURE 3A) [2]. The perfusion classification of uterine leiomyomas was regarded as type A uterine leiomyoma (the time signal intensity curve of uterine leiomyoma was less than that of myometrium) (FIGURE 3B) [3]. The patient was indicated single phase HIFU surgery under the guidance of magnetic resonance imaging by which clinician utilized 40 treatment cells (12 mm, n=12; $14 \mathrm{~mm}, \mathrm{n}=18 ; 16 \mathrm{~mm}, \mathrm{n}=10$ ) and a mean power of $158 \pm 26 \mathrm{~W}$. After 144 min of treatment duration, a mean nonperfused volume ratio of all leiomyomas was 98\% (FIGURE 3C). At 24 h post treatment, patient was discharged without adverse events. At 6 months of follow-up, all leiomyomas were shrunk with a total mean volume reduction ratio of $52 \%$ and the pelvic tension symptom was resolved entirely (FIGURE 3D). In addition, anti-mullerian hormone level prior to ablation and after 6 months of follow-up was preserved comprehensively at the level of $3.71 \mathrm{ng} / \mathrm{mL}$ [8].

A nulliparous 28 y old patient with history of leiomyoma suffered from urinary retention and pelvic pressure admitted to department 
of gynecology. On the physical examination, clinician found a big palpable mass on the pelvis. On T2-weighted image, there was an intramural leiomyoma located on the anterior wall of retroflexed uterus with a diameter of $151 \mathrm{~mm}$ considered as a type II leiomyoma (the signal intensity of leiomyoma was higher than that of skeletal muscle and lower than that of myometrium) (FIGURE 4A) [2]. On perfusion weighted image, leiomyoma was regarded as a type A leiomyoma (the time signal intensity curve of leiomyoma was lower than that of myometrium) (FIGURE 4B) [3]. The patient desired to undergo HIFU instead of open surgery. The HIFU surgery under the guidance of magnetic resonance imaging exploited 52 treatment cells $(12 \mathrm{~mm}, \mathrm{n}=8 ; 14 \mathrm{~mm}, \mathrm{n}=23$; $16 \mathrm{~mm}, \mathrm{n}=21$ ) placed with one layer strategy, and a mean power of $150 \pm 22 \mathrm{~W}$ yielded a nonperfused volume ratio of 91\% (FIGURE 4C) with a total treatment time of 232 minutes. At $12 \mathrm{~h}$ post ablation, patient was discharged without side effects. At 6 month follow-up, the diameter of leiomyoma was reduced to 82 $\mathrm{mm}$ and the complaint symptoms were resolved completely (FIGURE 4D). In addition, serum anti-mullerian concentration of this case prior to ablation and after 6 months of treatment was preserved entirely at the level of $3.21 \mathrm{ng} / \mathrm{mL}[8]$.

\section{Discussion}

HIFU was depended on the biological thermal principle of high intensity focused ultrasound on the targeted spot to lift the tissues' temperature up to the threshold of coagulative necrosis and protein denaturation (FIGURE 5) [2,3]. In a recent study by He et al., the findings revealed that HIFU was very effective in eradicating multiple leiomyomas of black women. In their study, the median number of uterine fibroids was 6 (3-9). The median non-perfused volume ratio was $85.2 \%$ with overall mean treatment time $97.3 \mathrm{~min} \pm 61.3$ (40-240) [9]. No major complications occurred during and after HIFU treatment. At 6 month follow-up, the overall mean shrinkage of 21 cases was about $52.5 \%$ \pm 36.3 . In this study, we also performed single phase HIFU flourishingly on patient with 15 leiomyomas without major adverse events fully agreement with a previous study [9].

In this study, we utilized "one layer strategy" treatment cells by which the heat accumulation

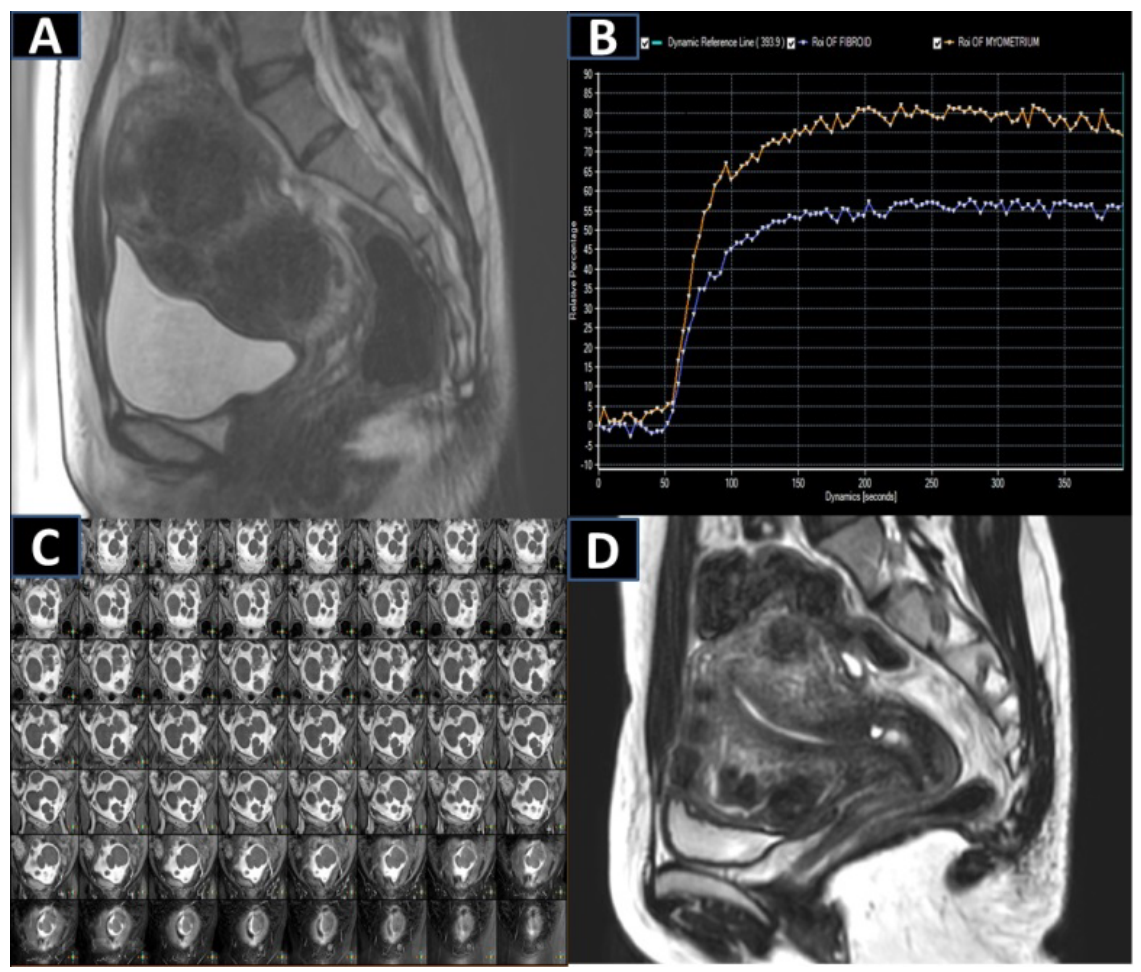

Figure 3. (A) Sagittal T2-weighted image at screening phase shows multiple uterine leiomyomas. (B) Axial perfusion-weighted image at screening phase shows the time signal intensity curve of uterine leiomyoma lower than that of myometrium. (C) Coronal contrast enhancement T1-weighted image after treatment shows near-complete ablation of all uterine leiomyomas. (D) Sagittal T2-weighted image at 6-month follow-up shows significant shrinkage of all uterine leiomyomas. 


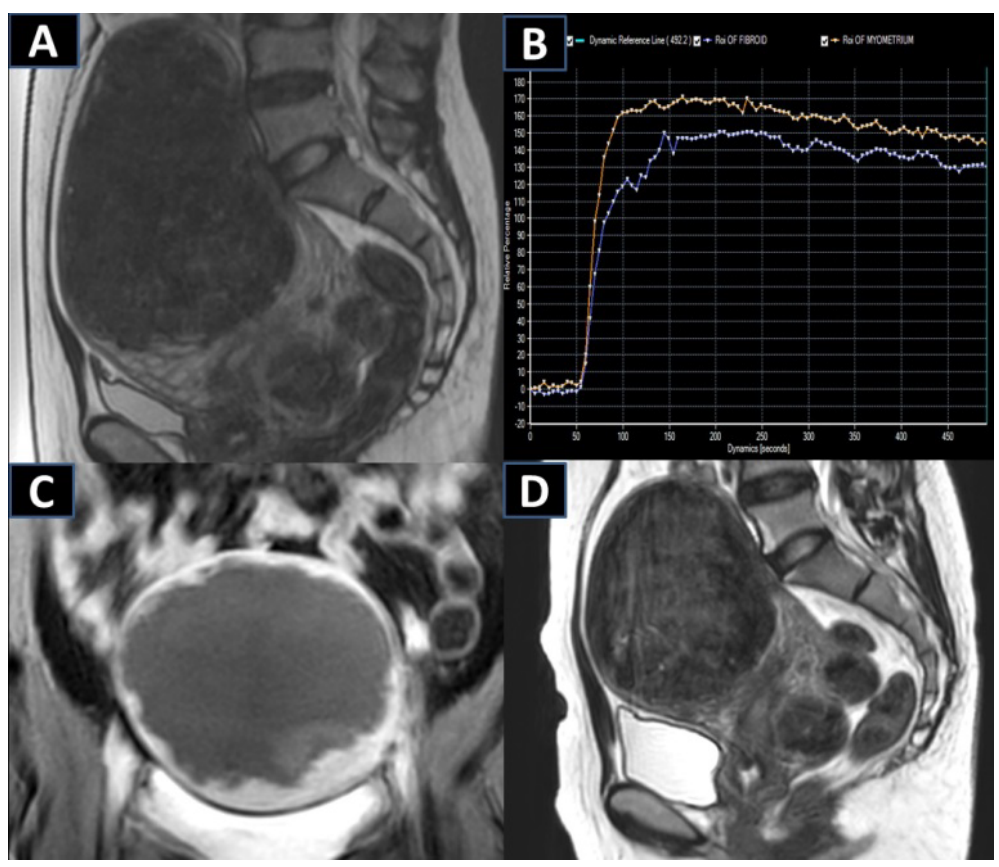

Figure 4. (A) Sagittal T2-weighted image at screening phase shows a huge uterine leiomyoma. (B) Axial perfusion-weighted image at screening phase shows the time signal intensity curve of uterine leiomyoma lower than that of myometrium. (C) Coronal contrast enhancement T1-weighted image after treatment shows near-complete ablation of uterine leiomyoma. (D) Sagittal T2-weighted image at 6-month follow-up shows significant shrinkage of uterine leiomyoma.

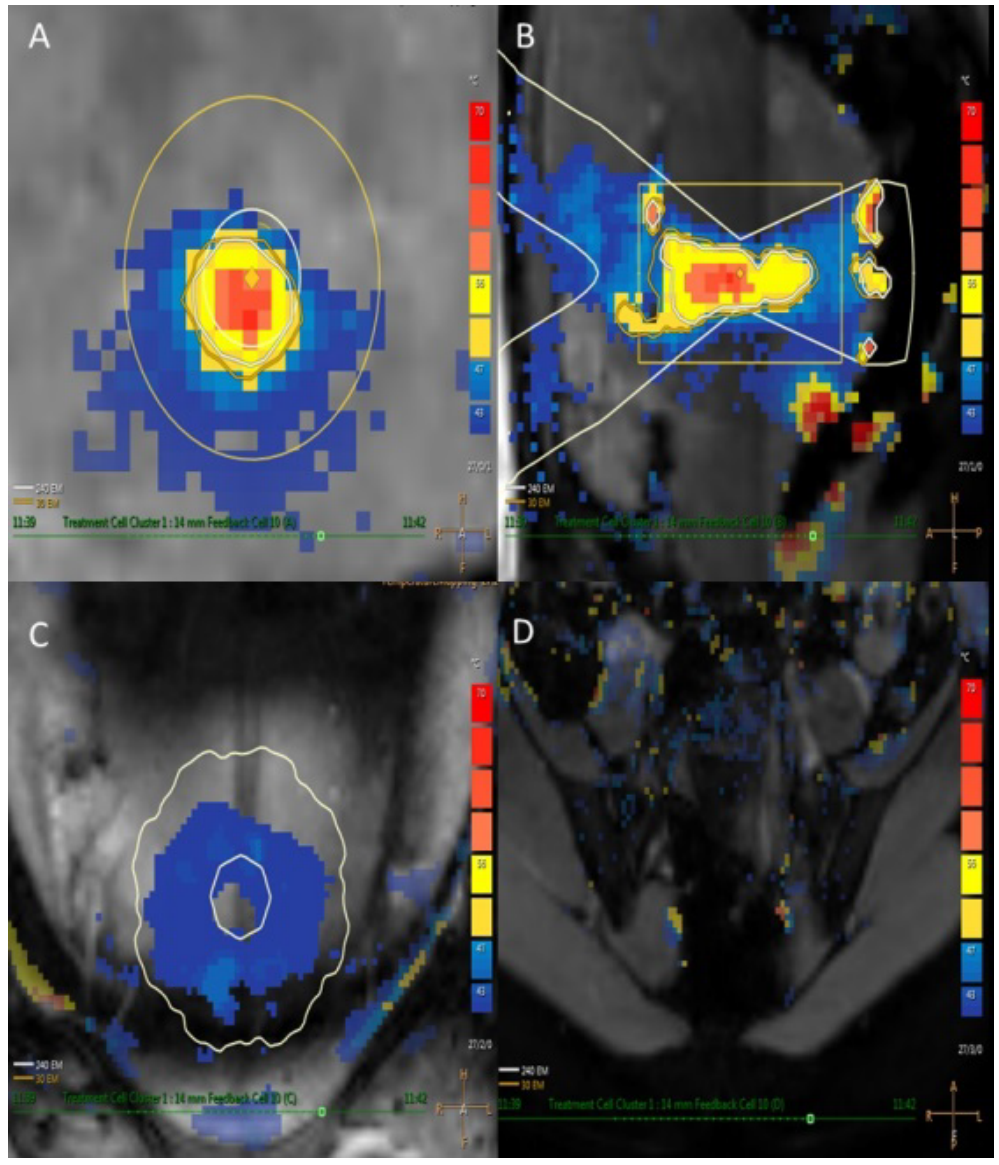

Figure 5. The temperature map images show (A) coronal plane, (B) sagittal plane, (C) near-field, and (D) far-field. 
in the nearfield of the focal plane can be taken advantage to ablate the huge leiomyoma with diameter $\geq 150 \mathrm{~mm}$. The plane for putting treatment cells was positioned at the anterior two-thirds of the tumors (FIGURE 6). The heat accumulation in the anterior part of tumors can enhance the treatment speed which will reduce the risk of deep vein thrombosis owing to long treatment time in prone position. In addition, this strategy ensures that two-thirds of the tumor automatically necrosis because of gradual heat accumulation phenomenon during the sonication procedure (FIGURE 7). The effectiveness of placing the treatment cells on the same plane for eradicating uterine fibroids with mean diameter $\geq 110 \mathrm{~mm}$ was demonstrated in two previous studies [10,11].

Nonetheless, a recent study found acute kidney damage due to tumor lysis statement after HIFU treatment on a $140 \mathrm{~mm}$ diameter uterine fibroid [12]. Therefore, in case of too large uterine fibroids, clinicians should consider to reduce the tumor diameter prior to HIFU treatment to ensure patient safety profile and improve ablation efficacy. It is concluded that size of uterine fibroids can differ from small tumor localized on the uterus to huge tumor conquered the whole abdominal cavity. Uterine fibroids are relied on estrogen to maintain and develop. One of the medications in treatment strategy is to reduce the concentration of serum estrogen. Gonadotropin-releasing hormone agonist ( $\mathrm{GnRH}$ agonist) is indicated in treatment of uterine fibroids by producing the hypoestrogen statement to inhibit the development of tumors. It is concluded that the mean volume reduction of uterine fibroids was $36 \%$ after an indication of 3 month GnRH

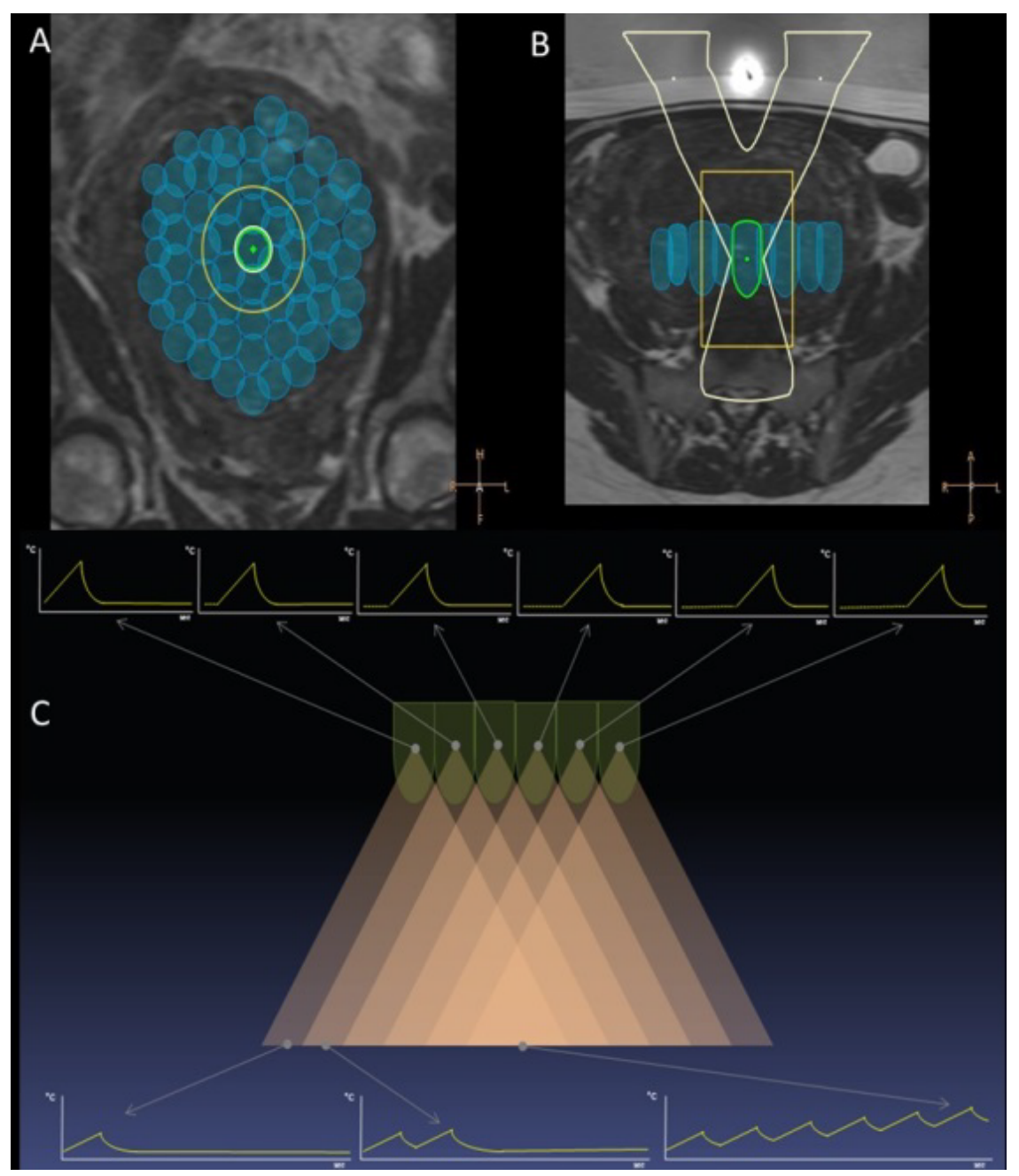

Figure 6. T2W images show one layer strategy with all treatment cells placed in the same (A) coronal plane and (B) axial plane. The center of the overlapped sonication area (C) manifests the gradual heat accumulation phenomenon. 


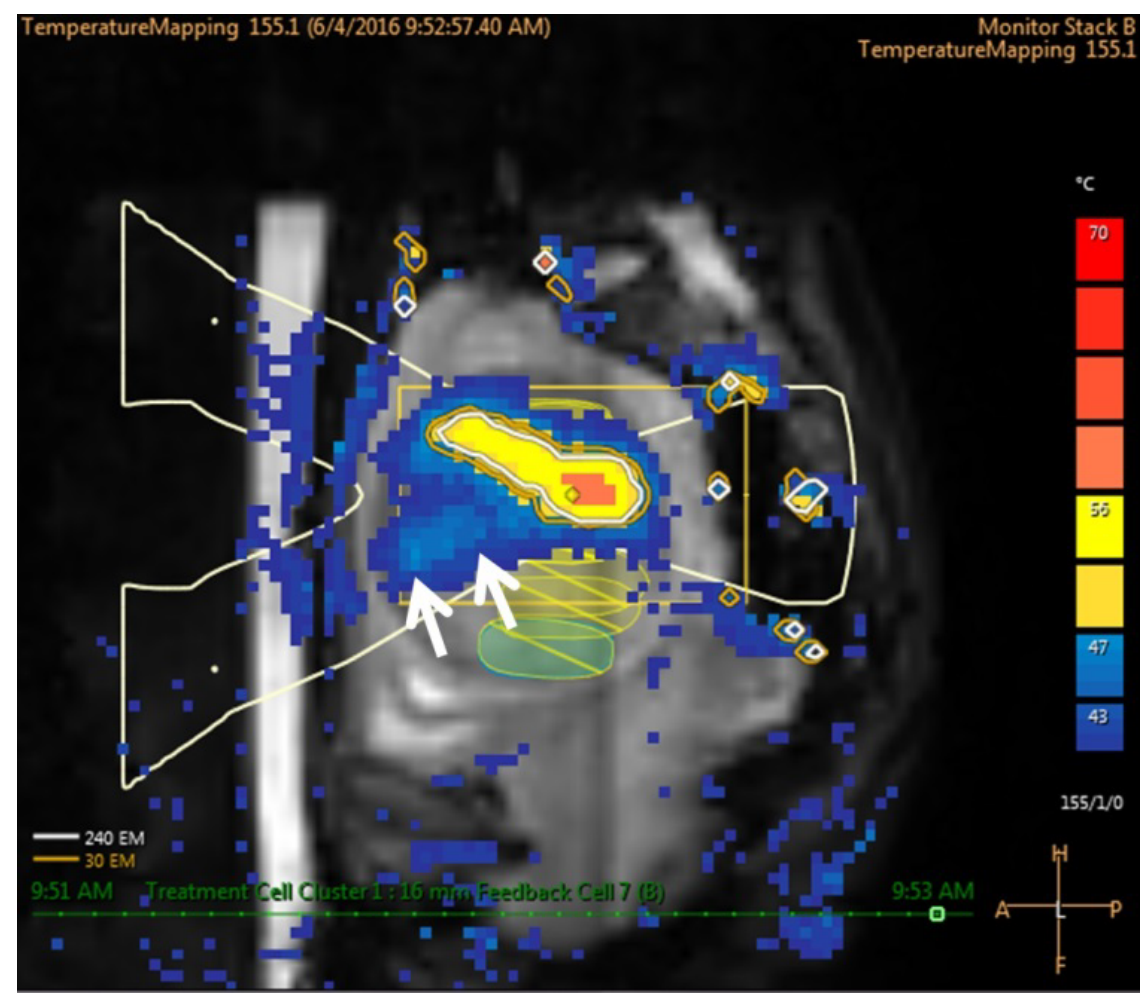

Figure 7. The one-layer strategy in the same sagittal plane shows heat accumulation in the anterior part of the tumor (white arrow).
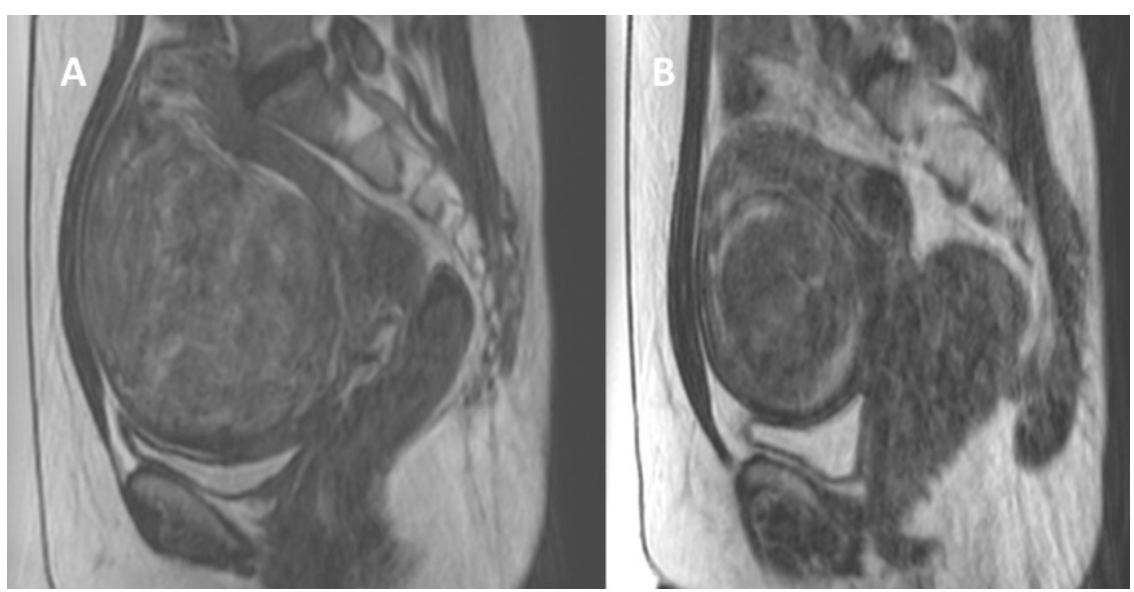

Figure 8. Sagittal T2W images show the uterine fibroid (A) prior to GnRH agonist therapy and (B) after 3 months of GnRH agonist therapy; the volume of uterine fibroid reduced up to $40 \%$.

agonist for the uterine fibroid diameter $\geq 10$ $\mathrm{cm}$, (FIGURE 8) [13]. In another study, uterine fibroid volume and perfusion are alleviated after utilizing 3 month GnRH agonist so that uterine fibroid tissue is more adapted to the HIFU ablation [14].

During the treatment process, utilizing continuously intravenous infusion oxytocin $(0.1 \mathrm{U} / \mathrm{min})$ can promote the mild contraction of the myometrium which limits the amount of blood to the uterus contributed to the efficiently cumulative heat at the targeted tissue during ablation procedure. Thus, it enhances the treatment speed and ablation efficacy in case of large uterine fibroids. It is also concluded that after the use of oxytocin, the wash-in rate and maximum signal intensity of uterine fibroids was significantly attenuated; simultaneously, timeto-peak and mean transit time of uterine fibroids were significantly perpetuated [15]. Hence, intravenous infusion of oxytocin can reduce significantly not only the average sonication energy but also the sonication time to attain $60^{\circ} \mathrm{C}$ for a focused point. Furthermore, the 
sonication energy to elevate tissue temperature $1^{\circ} \mathrm{C}$ is significantly lower than without utilizing infusion of oxytocin. As a result, oxytocin could significantly reduce the mean power during the ablation procedure, and enhance the treatment speed and ablation efficacy in case of large uterine fibroids [16].

\section{Conclusion}

According to the findings in this study, HIFU treatment should be considered as an alternative therapeutic method for patients with multiple leiomyomas and huge leiomyomas. Nevertheless, clinicians should optimize the treatment strategy in case of huge leiomyoma and multiple leiomyomas for ensuring the safety of patients. Further studies will be crucial to validate the efficacy and safety of HIFU on the large population of patients with multiple leiomyomas and huge leiomyoma.

\section{Disclosure statement}

Informed consent to all patients in this article was obtained. Nguyen Minh Duc and Huynh Quang Huy contributed equally to this article. All authors read and approved manuscript. The authors of this manuscript declared no conflict of interest.

\section{REFERENCES}

1. Stewart EA. Uterine fibroids. N. Engl. J. Med. 372: 1646-1655, (2015).

2. Funaki K, Fukunishi $\mathrm{H}$, Funaki $\mathrm{T}$ et al. Magnetic resonance-guided focused ultrasound surgery for uterine fibroids: relationship between the therapeutic effects and signal intensity of preexisting T2 weighted magnetic resonance images. Am. J Obstet. Gynecol. 196: 1-6, (2007).

3. Keserci B, Duc NM. The role of T1 perfusionbased classification in magnetic resonanceguided high-intensity focused ultrasound ablation of uterine fibroids. Eur. Radiol. 27: 5299-5308, (2017).

4. Haar G, Coussios C. High intensity focused ultrasound: physical principles and devices. Int. J. Hyperthermia. 23: 89-104, (2007).

5. Coussios CC, Farny CH, Haar GT et al. Role of acoustic cavitation in the delivery and monitoring of cancer treatment by highintensity focused ultrasound (HIFU). Int. J. Hyperthermia. 23: 105-120, (2007).

6. Hynynen K, Freund WR, Cline HE et al. A clinical, noninvasive, MR imaging-monitored ultrasound surgery method. Radiographics. 16: 185-195, (1996).

7. Kim YS. Advances in MR image-guided highintensity focused ultrasound therapy. Int. J. Hyperthermia. 31: 225-232, (2015).

8. Lee JS, Hong GY, Lee KH et al. Changes in anti-müllerian hormone levels as a biomarker for ovarian reserve after ultrasound-guided high-intensity focused ultrasound treatment of adenomyosis and uterine fibroid. BJOG. 124: 18-22, (2017).

9. He M, Jacobson $\mathrm{H}$, Zhang $\mathrm{C}$ et al. A retrospective study of ultrasound-guided high intensity focussed ultrasound ablation for multiple uterine fibroids in South Africa. Int. J. Hyperthermia. 5: 1-7, (2018).

10. Kim YS, Kim JH, Rhim $\mathrm{H}$ et al. Volumetric MR-guided high-intensity focused ultrasound ablation with a one-layer strategy to treat large uterine fibroids: initial clinical outcomes. Radiology. 263: 600-609, (2012).

11. Hou R, Wang L, Li S et al. Pilot study: safety and effectiveness of simple ultrasound-guided high-intensity focused ultrasound ablating uterine leiomyoma with a diameter greater than 10 cm. British. J. Radiol. 91: 102, (2018).

12. Park JH, Yang IM, Kim YH et al. High Intensity Focused Ultrasound Treatment-Induced Tumor Lysis Syndrome in Uterine Myoma Patient. Soonchunhyang Medical. Science. 21: 99-101, (2015).

13. Zaher S, Gedroyc WM, Regan L. Patient suitability for magnetic resonance guided focused ultra- sound surgery of uterine fibroids. Eur. J. Obstet. Gynecol. Reprod Biol. 143: 98-102, (2009).

14. Smart OC, Hindley JT, Regan L et al. Magnetic resonance guided focused ultrasound surgery of uterine fibroids: the tissue effects of $\mathrm{GnRH}$ agonist pre-treatment. Eur. J. Radiol. 59:163167, (2006).

15. Wang Y, Ren D, Wang W. The Influence of Oxytocin on the Blood Perfusion of Uterine Fibroids: Contrast-enhanced Ultrasonography Evaluation. J. Med. Ultrasound. 24: 13-17, (2016).

16. Huang X, He M, Liu YJ et al. Effect of oxytocin on uterine fibroids treated by ultrasound ablation (in Chinese). Zhonghua. Fu. Chan. Ke. Za. Zhi. 46: 412-415, (2011). 